\title{
Plasma cells in primary melanoma. Prognostic significance and possible role of IgA
}

Francesca M Bosisio ${ }^{1,2}$, James S Wilmott ${ }^{3}$, Nathalie Volders ${ }^{1}$, Marjorie Mercier ${ }^{4}$, Jasper Wouters ${ }^{1}$, Marguerite Stas ${ }^{5}$, Willeke AM Blokx ${ }^{6}$, Daniela Massi ${ }^{7}$, John F Thompson ${ }^{3}$, Richard A Scolyer ${ }^{3,8}$, Nicolas van Baren ${ }^{4}$ and Joost J van den Oord ${ }^{1}$

${ }^{1}$ Laboratory of Translational Cell and Tissue Research, University of Leuven, KUL, Leuven, Belgium;

${ }^{2}$ Università Degli Studi di Milano-Bicocca, Milan, Italy; ${ }^{3}$ Melanoma Institute Australia, The University of Sydney, Sydney, NSW, Australia; ${ }^{4}$ Ludwig Institute for Cancer Research and de Duve Institute, Université Catholique de Louvain, Brussels, Belgium; ${ }^{5}$ Department of Surgical Oncology, UZ Gasthuisberg and KU Leuven, Leuven, Belgium; ${ }^{6}$ Radboud University Medical Center, Nijmegen, The Netherlands; ${ }^{7}$ Department of Surgery and Translational Medicine, University of Florence, Florence, Italy and ${ }^{8}$ Department of Tissue Pathology and Diagnostic Oncology, Royal Prince Alfred Hospital, Camperdown, NSW, Australia

Melanoma is not only one of the most immunogenic cancers but also one of the most effective cancers at subverting host immunity. The role of $\mathrm{T}$ lymphocytes in tumor immunity has been extensively studied in melanoma, whereas less is known about the importance of B lymphocytes. The effects of plasma cells (PCs), in particular, are still obscure. The aim of this study was to characterize pathological features and clinical outcome of primary cutaneous melanomas associated with PCs. Moreover, we investigated the origins of the melanoma-associated PCs. Finally, we studied the outcome of patients with primary melanomas with PCs. We reviewed 710 melanomas to correlate the presence of PCs with histological prognostic markers. Immunohistochemistry for CD138 and heavy and light chains was performed in primary melanomas (PM) and in loco-regional lymph nodes (LN), both metastatic and not metastatic. In three PM and nine LN with frozen material, VDJ-rearrangement was analyzed by Gene Scan Analysis. Survival analysis was performed on a group of 85 primary melanomas $>2 \mathrm{~mm}$ in thickness. Forty-one cases (3.7\%) showed clusters/sheets of PCs. PC-rich melanomas occurred at an older age and were thicker, more often ulcerated and more mitotically active $(P<0.05)$. PCs were polyclonal and often expressed IgA in addition to IgG. In LN, clusters/sheets of IgA+ PCs were found both in the sinuses and subcapsular areas. Analysis of VDJ-rearrangements showed the IgA to be oligoclonal. Melanomas with clusters/sheets of PCs had a significantly worse survival compared with melanomas without PCs while, interestingly, melanomas with sparse PCs were associated with a better clinical outcome $(P=0.002)$. In conclusion, melanomas with sheets/clusters of PCs are associated with worse prognosis. IgG and IgA are the isotypes predominantly produced by these PCs. IgA oligoclonality suggests an antigen-driven response that facilitates melanoma progression by a hitherto unknown mechanism.

Modern Pathology (2016) 29, 347-358; doi:10.1038/modpathol.2016.28; published online 12 February 2016

Melanoma is not only one of the most immunogenic cancers but also one of the cancers that is most adept at circumventing host antitumor immunity. ${ }^{1}$ In fact, the phenomenon of regression in primary melanomas is frequently only partial $(23-58 \%)$, and complete regression is rare $(12.4 \%)$, suggesting the presence of mechanisms that allow the tumor to

Correspondence: Dr FM Bosisio, MD, Laboratory of Translational Cell and Tissue Research, University of Leuven, KUL, Minderbroedersstraat 12 blok p - bus 1032, Leuven 3000, Belgium.

E-mail: f.bosisio1@gmail.com

Received 18 September 2015; revised 13 November 2015; accepted 21 December 2015; published online 12 February 2016 resist immune attack. ${ }^{2}$ In recent years, the immune response in melanoma has gained much attention owing to the efficacy of new therapies that act on its modulation by blocking immune check points. ${ }^{3-7}$ The immune response is the result of complex interactions between different types of cells, which can result in an immune-stimulatory signal as well as an immune-suppressive signal. Moreover, the very same type of cell can be influenced by the microenvironment to polarize into different functional states according to the need for an active immune response or of immune tolerance at a certain moment. This has been demonstrated for macrophages (M1 and M2 polarization), ${ }^{8-11}$ 
neutrophils (N1 and N2 polarization) ${ }^{12,13}$ and T lymphocytes (Th1, Th2, Th17 responses). ${ }^{14-16}$

The role of $\mathrm{T}$ lymphocytes and macrophages has been extensively studied in melanoma, ${ }^{13,17-22}$ whereas less is known on the role of B lymphocytes and plasma cells (PCs). Recently, B lymphocytes have turned out to constitute between 0 and $50 \%$ of the tumor-infiltrating lymphocytes, ${ }^{23-25}$ but controversy exists as to their precise role in melanoma progression. ${ }^{25-28}$ Furthermore, the role of PCs, the terminally differentiated form of B lymphocytes, has been the subject of two studies, one that links them with an increased risk of lymph node metastasis ${ }^{29}$ and one that shows an association with poor survival, ${ }^{30}$ but the mechanisms underpinning these observations remain obscure.

The aim of the current study was to identify melanomas with a significant PC component in the inflammatory infiltrate, to study their detailed clinico-pathological characteristics and immunoglobulin expression and to speculate on the origin of the $\mathrm{PC}$ response by examining the melanoma-draining loco-regional lymph nodes.

\section{Materials and methods}

\section{Evaluation of Cases}

Seven hundred and ten consecutive cases of invasive primary cutaneous melanoma from the Department of Pathology of the University Hospitals Leuven (KU Leuven), Belgium, were reviewed. According to their subtype, there were 544 superficial spreading melanomas (SSM), 83 nodular melanomas (NM), 25 acral lentiginous melanomas (ALM) and 58 'others' category that included special variants, such as nevoid or desmoplastic melanomas, or cases with unknown subtype. The age of the patients ranged from 11 to 97 years (median: 63 years). In all, 417 were females, and 293 males. Also, 283 melanomas occurred on the limbs, 213 on the trunk, 83 in the head and neck area and 38 on acral sites; from 93 cases, the site was not known. All lesions were evaluated for histological prognostic factors. In addition, 15 primary melanomas from Radboud University Medical Center (Nijmegen, The Netherlands) and 6 cases from the University of Florence (Florence, Italy), all containing sheets of PCs, were available for analysis. Melanoma cases were semiquantitatively evaluated according to the quantity of PCs in the inflammatory infiltrate surrounding the melanoma and subdivided into four groups (Figure 1a). The first group included melanomas without PCs ( $\mathrm{PC}^{\text {neg }}$ ), while the other three groups included melanomas with increasing numbers of PCs (in general, PC+).

All the available slides for each case were screened for the presence of PCs. The relative number of PCs was quantified by a scoring system taking both the number and distribution pattern (clusters or single cells) into consideration. Score 1 was assigned when the PCs in the infiltrate were sparse and scattered, with no tendency to cluster (PC1). Score 2 was used for melanomas in which PCs were more abundant, with a tendency to group in discrete clusters, containing at least five PCs in contact with each other (PC2). The score 3 category showed confluent clusters of PCs, forming sheets (PC3). To evaluate the impact of PCs on the histology and outcome of melanoma, we grouped scores PC2 and PC3 under the heading "plasma cell-rich melanomas' (PC-rich, 34 cases). In 14 cases of $\mathrm{PC}+$ melanoma, loco-regional lymphadenectomy specimens containing both involved lymph nodes (8 cases) and uninvolved lymph nodes (6 cases) were available for study.

To evaluate the outcome of PC+ melanomas, an additional data set of 85 patients with thick melanomas ( $>2 \mathrm{~mm}$, range $2.1-47 \mathrm{~mm}$ ) and clinical follow-up data from a minimum of 3 to a maximum of 12 years (range: 1099-4633 days) was collected from the files of the Melanoma Institute Australia, Sydney, and the Department of Tissue Pathology and Diagnostic Oncology, Royal Prince Alfred Hospital, Camperdown, New South Wales, Australia. We included this series of thick melanomas in order to enrich the samples for PC + melanomas and to minimize the impact of Breslow thickness on the outcome. In this melanoma set, the prevalent subtype was NM (31 cases), followed by SSM (24 cases) and ALM (12 cases). Eighteen cases were from special subtypes, mostly desmoplastic melanomas and malignant blue nevus cases. The most frequent site of occurrence was head and neck (26 cases), followed by trunk (16 cases), acral sites (16 cases), and limbs (15 cases). The outcome of the patients included 36 cases alive without recurrence, 3 alive with melanoma, 20 dead from melanoma, 3 cases dead owing to unrelated cause and 11 cases dead from unknown cause.

\section{Immunohistochemistry}

All biopsies were fixed in 10\% buffered formalin and embedded in paraffin. The most representative section for each case was used for immunohistochemistry. Immunohistochemical analyses were performed on routinely fixed paraffin-embedded tissue sections on the Leica Bondmax automatic immunostainer using ready-to-use antibodies from Dako directed against CD138, IgG, IgA, IgM, IgD, IgE, CD3, CD20 and CD21. The peripheral nodal addressin antibody was from Sigma-Aldrich (peripheral nodal addressin (PNAd), clone: MECA, dilution 1:3000).

A semiquantitative score was used to evaluate the number of IgA + PCs relative to the total number of CD138+ PCs in the inflammatory infiltrate in sections stained with double IgA (red)/CD138 (brown) immunohistochemistry. The quantity of double IgA/CD138-positive PCs was scored as ;' -' if $<10 \%$ of CD138+ PCs was IgA+, '+' if IgA+/CD138 PC were 

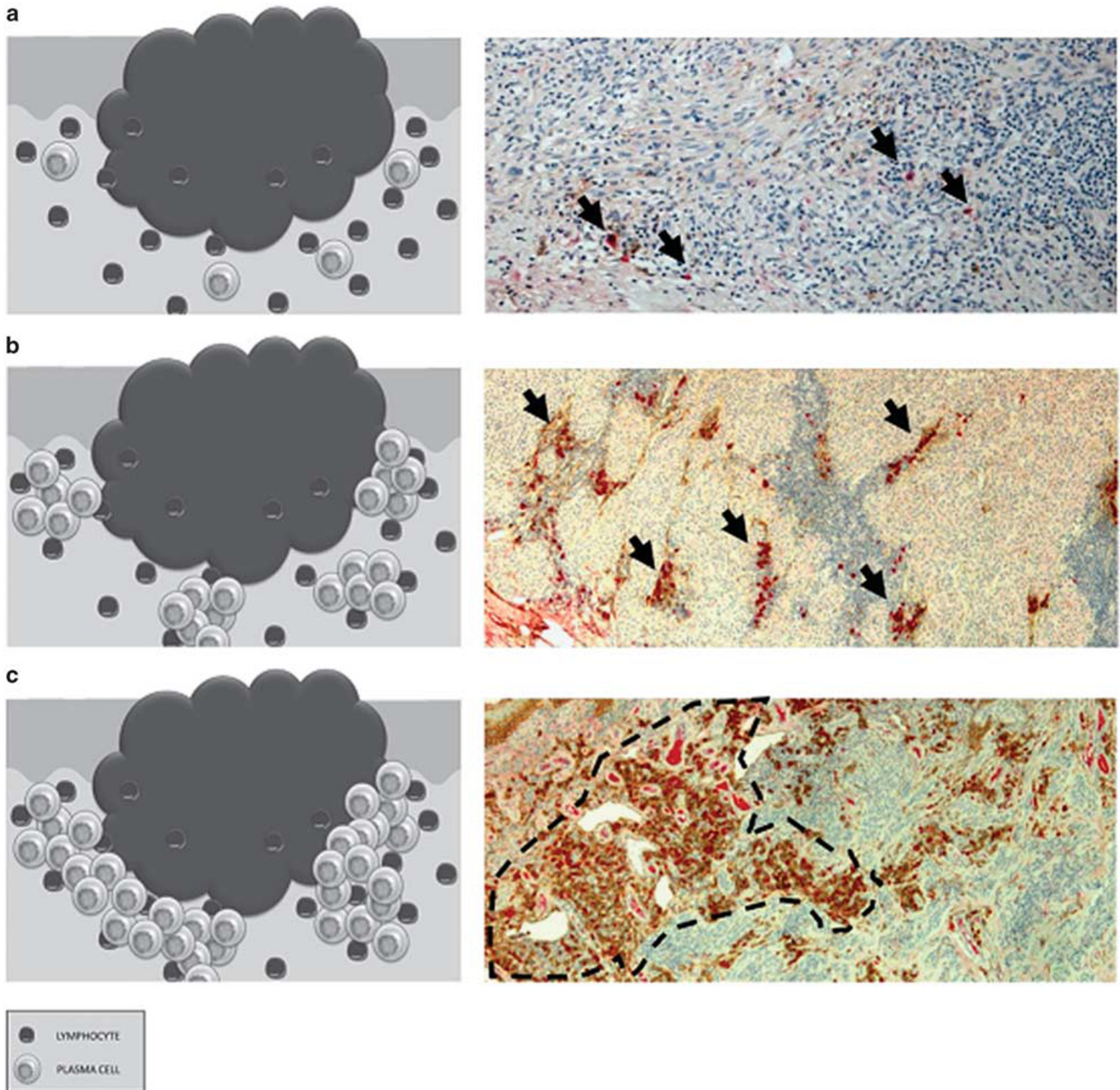

Figure 1 Definition of the plasma cells and IgA scores. (a) PC1 score was assigned when scattered plasma cells were found isolated in the infiltrate (arrows: plasma cells; double immunostaining for CD138 (brown) and IgA (red), × 10). (b) In PC2, plasma cells aggregated in clusters are found in the infiltrate (arrows: clusters of plasma cells; double immunostaining for CD138 (brown) and IgA (red), $\times 10$ ). (c) Large clusters of PCs merge together to form sheets in the tumoral stroma were scored as PC3 (a sheet is contoured by the discontinuous line; double immunostaining for CD138 (brown) and IgA (red), $\times 10$ ). IgA+CD138+ plasma cells were qualitatively quantified in the infiltrate as $<10 \%(\times 20)(\mathbf{d})$, between 10 and $50 \%(\times 20)(\mathbf{e})$, and $>50 \%$ or more of the total number of plasma cells $(\times 20)(\mathbf{f})$.

between 10 and $50 \%$ and ' ++ ' if $\geq 50 \%$ of the CD138+ PCs was IgA+ (Figure 1).

\section{Gene Scan Analysis}

Representative material, snap frozen in liquid nitrogen-cooled isopentane, was available from 2 PC+ primary melanomas and 14 lymph nodes draining PC+ melanomas. Total RNA from the tumor samples was isolated with the Total RNA and DNA Purification Kit from Macherey-Nagel, according to the manufacturer's protocol. Five hundred micrograms of RNA was reversely transcribed into cDNA using Superscript II reverse transcriptase (Invitrogen) and a random hexamer (Fermentas). Afer treatment with RNaseH (Fermentas), the resulting cDNA was diluted for a final volume of $40 \mu \mathrm{l}$. For the Ig heavy chain characterization of the samples, a set of primers specific for the secretory (s) type and the membrane 
d

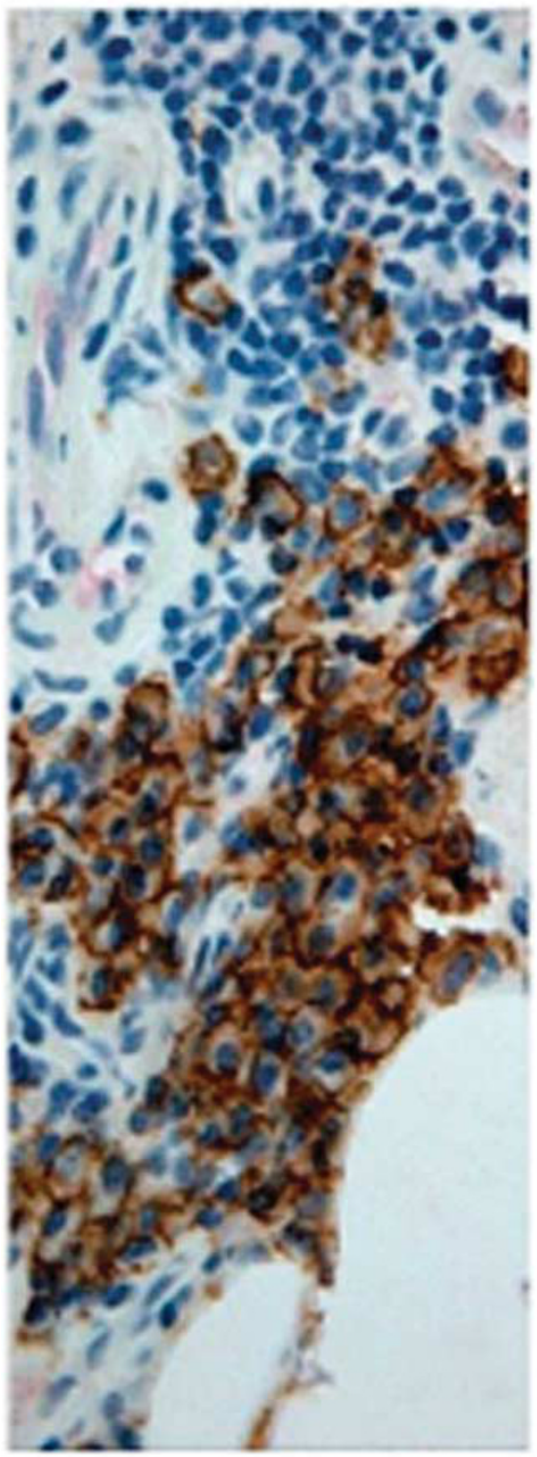

e

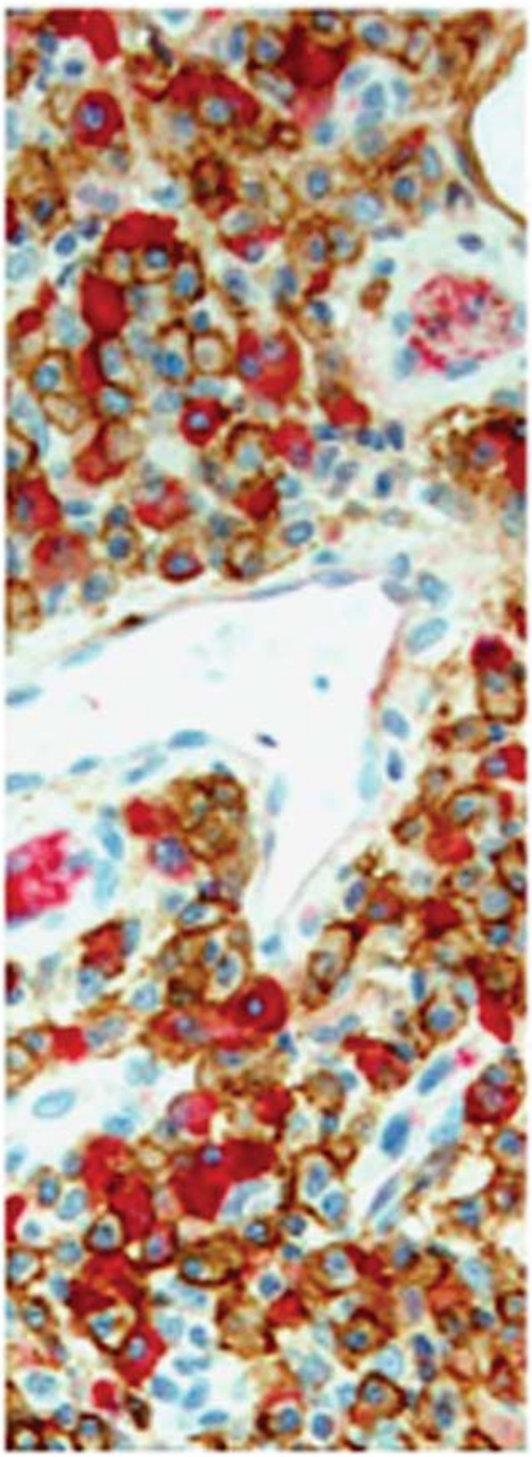

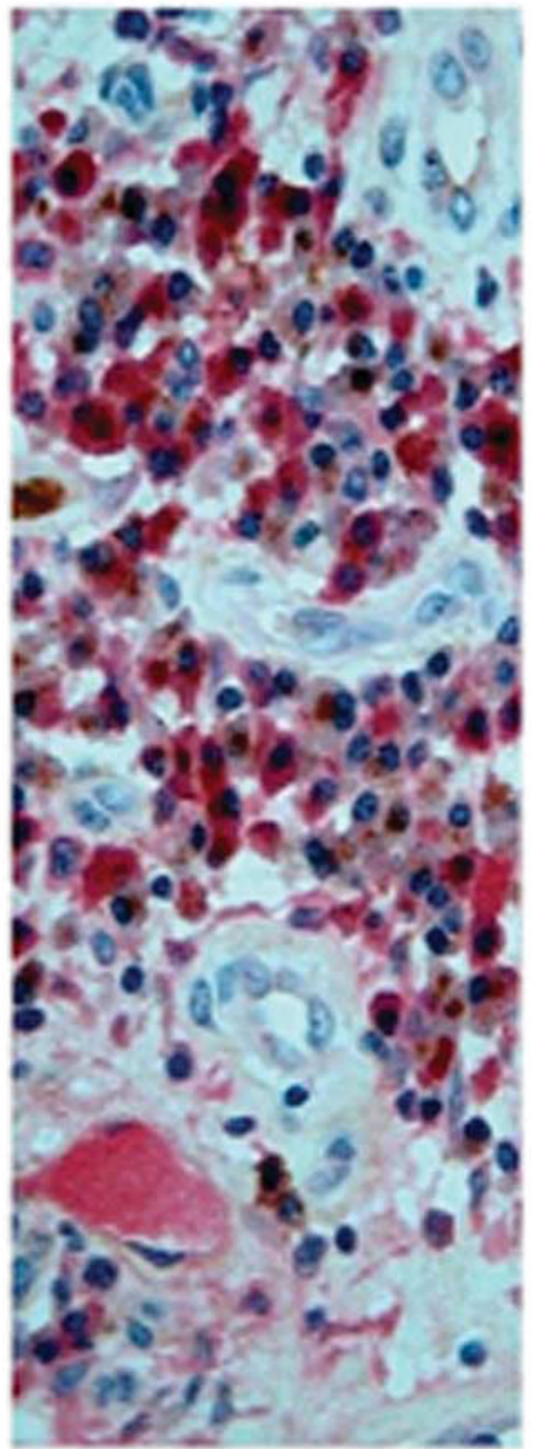

Figure 1 Continued

(m) type of all the immunoglobulins isotypes was used to perform the PCRs. Primers for actin and GADPH were used to check the cDNA quality, and a sample with water served as negative control. Amplification was carried out with Taq DNA polymerase (Takara), with $2.5 \mu \mathrm{l}$ of cDNA in a $25-\mu \mathrm{l}$ volume, and started with $4 \mathrm{~min}$ at $94^{\circ} \mathrm{C}$, followed by 36 cycles of $45 \mathrm{~s}$ at $94{ }^{\circ} \mathrm{C}, 45 \mathrm{~s}$ at $58^{\circ} \mathrm{C}, 1 \mathrm{~min}$ and $30 \mathrm{~s}$ at $72^{\circ} \mathrm{C}$, and ended by a final step of $5 \mathrm{~min}$ at $72^{\circ} \mathrm{C}$. The amplification products were checked with gel electrophoresis. For the Gene Scan Analysis, the cDNA was amplified by two rounds of nested PCR. All the primers used are listed in Supplementary Table S2. For the first PCR, amplification was carried out with $2.5 \mu \mathrm{l}$ of cDNA in a $25-\mu \mathrm{l}$ volume and started with $4 \mathrm{~min}$ at $94^{\circ} \mathrm{C}$, followed by 30 cycles of $45 \mathrm{~s}$ at $94^{\circ} \mathrm{C}, 45 \mathrm{~s}$ at $50^{\circ} \mathrm{C}$, $45 \mathrm{~s}+2 \mathrm{~s} /$ cycle at $72{ }^{\circ} \mathrm{C}$, for a total of 30 cycles, to end with $10 \mathrm{~min}$ at $72^{\circ} \mathrm{C}$. For the second PCR, amplification was carried out with $2.5 \mu \mathrm{l}$ of the first
PCR product diluted one in a hundred in a $25-\mu \mathrm{l}$ volume and started with $4 \mathrm{~min}$ at $94^{\circ} \mathrm{C}$, followed by 36 cycles of $45 \mathrm{~s}$ at $94^{\circ} \mathrm{C}, 45 \mathrm{~s}$ at $57^{\circ} \mathrm{C}, 45 \mathrm{~s}+2 \mathrm{~s} /$ cycle at $72{ }^{\circ} \mathrm{C}$, for a total of 30 cycles, to end with $10 \mathrm{~min}$ at $72{ }^{\circ} \mathrm{C}$. The amplification products were checked with gel electrophoresis. Size and quantity of the amplified DNA fragments were analyzed in combination with the Internal Standard Lane 600 (Promega) through the ABI PRISM 310 Genetic Analyzer and the Gene Scan Analysis. A polyclonal control (tonsil) and a monoclonal control (IgA myeloma cell line) were loaded together with the samples.

\section{Statistical Analysis}

Statistical analysis was performed with Microsoft Office Excel and the GraphPad Prism 6.01 software. The Mann-Whitney test for not normally distributed 
a
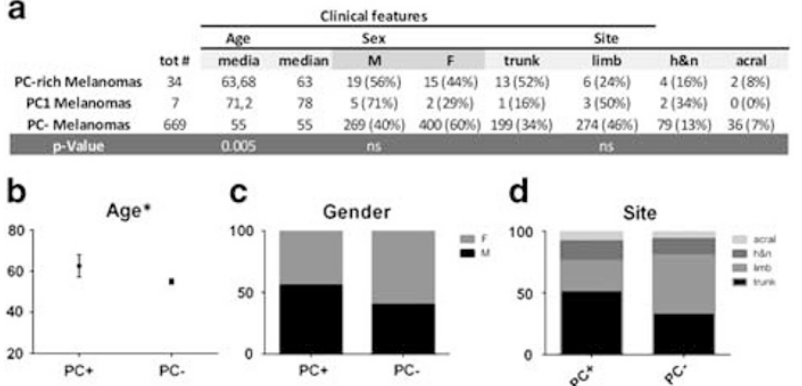

e

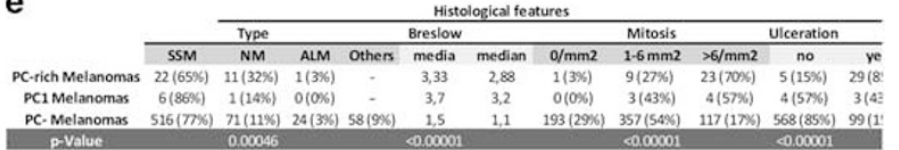

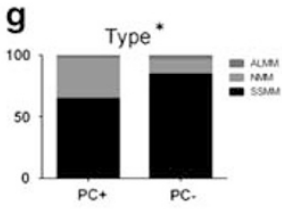

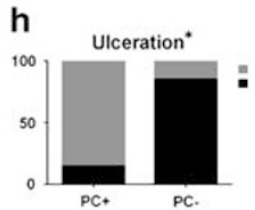

i

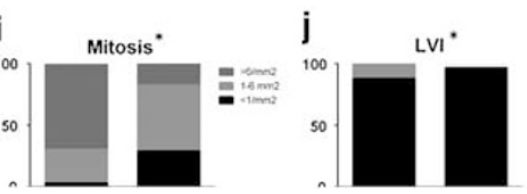

Figure 2 Statistical analysis of the clinical (a-d) and histopathological (e-j) features of PC-rich, PC1 and PC- melanomas. Parameters that reach significance are marked by asterisks. ALM, acral lentiginous melanoma; H\&N, head and neck; LVI, lymph/vascular invasion; NM, nodular melanoma; SSM, superficial spreading melanoma.

Table 1 Immunohistochemical analysis

\begin{tabular}{lccc}
\hline Case no. & PC score & IgA+/CD138+ & TLS \\
\hline 1 & 3 & $>50 \%$ & Yes \\
2 & 3 & $>50 \%$ & No \\
3 & 3 & $10-50 \%$ & No \\
4 & 3 & $10-50 \%$ & No \\
5 & 3 & $10-50 \%$ & No \\
6 & 3 & $<10 \%$ & No \\
7 & 2 & $>50 \%$ & No \\
8 & 2 & $10-50 \%$ & No \\
9 & 2 & $10-50 \%$ & No \\
10 & 2 & $10-50 \%$ & No \\
11 & 2 & $10-50 \%$ & No \\
12 & 2 & $<10 \%$ & No \\
13 & 1 & $<10 \%$ & Yes \\
14 & 1 & $<10 \%$ & No \\
15 & 1 & $<10 \%$ & No \\
16 & 1 & $<10 \%$ & No \\
& 1 & & \\
\hline
\end{tabular}

Double staining for IgA and CD138 showed an increase of the amount of IgA+ plasma cells that goes together with the quantity of plasma cells in the infiltrate. Immunohistochemistry for CD3, CD20, CD21 and PNAd identified the presence of tertiary lymphoid structures in only $2 / 16$ cases.

data was used to calculate the $P$-values for age and Breslow. The Chi-square test adjusted for not normally distributed data was used to evaluate the $P$-values for the correlation of the other clinical and histological parameters analyzed with the presence of PCs. Survival rates were calculated according to the Kaplan-Meier method.

\section{Results}

\section{Clinico-Pathological Features of PC+ Melanomas}

Of the 710 primary cutaneous melanomas from the University Hospital Leuven, 41 (3.7\%) showed a PC component in the inflammatory infiltrate associated with the melanoma. According to the PC score,
7 (18\%) showed scattered PCs (score PC1), 24 (58\%) had discrete clusters of PCs (score PC2) and 10 $(24 \%)$ contained sheets of PCs (score PC3). As PC1 melanomas represent a condition in which the quantity of PC is low and could be not biologically relevant, we focused on PC2 and PC3 cases to better characterize melanomas in which the amount of PC is important and labeled these 34 cases (82\%) as 'PC-rich' melanomas in statistical analysis. From score PC1 to score PC3, an increase in the number of intra-tumoral PCs that were in direct contact with the melanoma cells was observed. In fact, while in 6 out of the 7 PC1 melanomas the PCs were only in peritumoral location, PC2 and PC3 melanomas showed the PCs to be located within the tumor mass $(15 / 24(62.5 \%)$ and $8 / 10(80 \%)$ cases with intratumoral PCs, respectively, $P=0.006)$. Clinical and histological features of PC-rich melanomas are shown in Supplementary Table S1, and the statistical analysis is resumed in Figure 2. The average age at the diagnosis for PC-rich melanomas was 63 years (range 20-86, median 63) and 55 years for melanomas without PC (range 11-97, median 55), suggesting that PC-rich melanomas occur in patients 10 years older than $P^{\text {neg }}$ melanomas $(P=0.005)$. In all, 19/34 (56\%) patients with PC-rich melanomas were males, and 15/34 (44\%) were females. PC-rich melanomas affected, in decreasing order, the trunk, limbs, head and neck areas and acral sites, while $\mathrm{PC}^{\text {neg }}$ melanomas were mostly on the limbs and less frequently on the trunk, head and neck area and acral sites. PC-rich melanomas were more often of the nodular subtype than $\mathrm{PC}^{\text {neg }}$ melanomas $(P=0.00046)$. Three strongly evidence-based markers of bad prognosis in melanoma (high Breslow thickness, $>6$ mitoses $/ \mathrm{mm}^{2}$ and the presence of ulceration) were found to be associated with the presence of PCs in melanoma $(P<0.0001)$. The incidence of $\mathrm{PC}+$ melanomas increased to $19.5 \%$ if only cases with a Breslow thickness $>2 \mathrm{~mm}$ were taken into account. A 
correlation with the presence of lymphatic/vascular invasion was also found $(P=0.015)$. Histological examination of the 15 cases from Nijmegen, The Netherlands and 6 cases from Firenze, Italy revealed similar unfavorable prognostic features in melanomas with sheets of PC.

No significantly different clinical features were found between score PC1, PC2 and PC3. The three PC scores differed significantly only for two histopathological parameters, ie, the PC distribution in the tumor microenvironment and the thickness of the melanoma according to Breslow. Regarding the distribution of PCs, the number of intratumoral PCs increased from score PC1 to score PC3, indicating that, with increasing numbers of PCs in the inflammatory infiltrate, these PC tended to be more in direct contact with melanoma cells $(P=0.006)$. Moreover, PC3 melanomas were significantly thicker than PC2 and PC1 melanomas $(P=0.008)$. All other histopathological parameters (subtype, mitotic rate, ulceration, regression, lymphatic/vascular invasion, microsatellites) were not significantly correlated with the number of PCs in the infiltrate.

\section{Immunohistochemical Features of PC+ Melanomas}

Sixteen cases of PC+ primary melanomas were available for immunohistochemical analysis. Immunohistochemistry for Ig light chains showed the PCs to be polyclonal (data not shown) and staining for different isotypes of immunoglobulins (IgA, IgG, $\operatorname{IgM}, \operatorname{IgD}, \operatorname{IgE}$ ) revealed that the major part (usually $>50 \%$ ) of the PCs in the melanoma-associated inflammatory infiltrate was positive for IgG (data not shown). Interestingly, an equally important PC component of the infiltrate expressed IgA. Using double chromogenic immunohistochemistry for the membranous PC marker CD138 (brown) and cytoplasmic IgA (red), the quantity of IgA+ PCs as a fraction on the total of the CD138+ PCs was evaluated in PC+ melanomas. In 3/16 (18\%) of the cases, $>50 \%$ of PCs expressed IgA, in $7 / 16(44 \%), 10-50 \%$ of PCs expressed IgA and 6/16 (38\%) cases were devoid of a significant IgA+ PC component. In total, $56 \%$ of cases showed $>10 \%$ of all PCs to express IgA, and all these cases had clusters (PC2 score) or sheets (PC3 score) of PCs. The distribution of the IgA+CD138+ score from PC1 to PC3 melanomas showed that IgA+ PCs increased with increasing numbers of PCs in the melanoma (Table 1). In order to rule out that ulceration by itself was the trigger for IgA switch, 20 randomly chosen non-neoplastic ulcers and two (positive control) genital and mucosal ulcers were analyzed histologically and immunohistochemically. Nine had PCs in the infiltrate, and these ulcers occurred more frequently in the head and neck areas (Supplementary Table S3). On double chromogenic immunohistochemistry, the number of IgA+ PCs was generally low in cutaneous non-neoplastic ulcers as compared with those at mucosal sites where equal numbers of IgA+ and IgG+ PCs were found.

As they represent a site of PC formation, the occurrence of tertiary lymphoid structures (TLS) in the inflammatory stroma was investigated by immunostainings in consecutive serial sections of 16 cases for CD20 (B cells), CD3 (T cells), CD21 (follicular dendritic cells (FDC)) and PNAd, a high endothelial venule (HEV) marker. The presence of $\mathrm{B}$ and $\mathrm{T}$ cells, as well as FDC and HEV together in a lymphoid aggregate, was considered to be sufficient to define a TLS. ${ }^{31}$ According to this criterium, only 2 out of the 16 cases of PC+ melanoma presented with TLS (Table 1).

\section{Molecular Analysis of Ig Genes Expressed by PC+ Melanomas}

We first analyzed the isotype of the immunoglobulinexpressing cells present in two PC-rich primary melanomas, for which frozen material was available. We used RT-PCR to detect the IGHD, IGHM, IGHG, IGHA and IGHE transcripts, which encode the constant regions of Ig heavy chains (Figure 3a). The secreted isoforms of IgG and IgA were more expressed than both the corresponding membranous isoforms and other isotypes, thus confirming that they are the main isotypes expressed by PCs in melanoma.

To further characterize the clonality of the infiltrating PCs, we analyzed the diversity of the Ig gene repertoire with the immunoscope approach. To this aim, we used two rounds of nested RT-PCR, including fluorochrome-coupled primers for the last PCR round, to amplify all the VDJ sequences present in IgG-s and IgA-s transcripts. The amplified fluorescent fragments were then separated according to their length by high-resolution electrophoresis (Figure $3 \mathrm{~b}$ ). In both tumors, the IgG were found to have a polyclonal pattern, whereas the IgA repertoire was more restricted, indicating oligoclonality.

\section{Analysis of Lymph Nodes Draining PC+ Melanomas}

In the loco-regional lymph nodes, the same score used for primary melanomas was applied to quantify the amount of PC infiltrates. In lymph nodes with score 1, scattered PCs were predominantly localized in the medullary sinuses. In lymph nodes with score 2 and 3 , PC $^{\text {neg }}$ clusters and sheets were found in the medullary sinuses as well as in the sub-capsular region. In 12/14 lymph nodes draining the site of a PC+ melanoma, the PC score in the lymph node was the same or higher than the score in the corresponding primary melanoma. Therefore, a good correlation existed between the PC score in draining lymph nodes and primary melanoma (Figure 4). Using double IgA/CD138 immunohistochemistry, the percentage of IgA+PC varied between 10 and $50 \%$ of the total number of nodal PCs in 11 out of the 14 cases; moreover, a high percentage of 
a

Case \# IgG-m IgG-s IgD-m IgD-s IgM-m IgM-s IgA-m IgA-s IgE-m IgE-s

\begin{tabular}{ccccccccccc}
\hline & + & +++ & $+/-$ & $+/$ & + & ++ & + & ++ & - & $+/-$ \\
2 & ++ & +++ & + & ++ & + & ++ & ++ & +++ & $+/-$ & + \\
IgA control & $/$ & $/$ & $/$ & $/$ & $/$ & $/$ & +++ & +++ & $/$ & $/$ \\
IgE control & $/$ & $/$ & $/$ & $/$ & $/$ & $/$ & $/$ & $/$ & ++ & +++
\end{tabular}

b

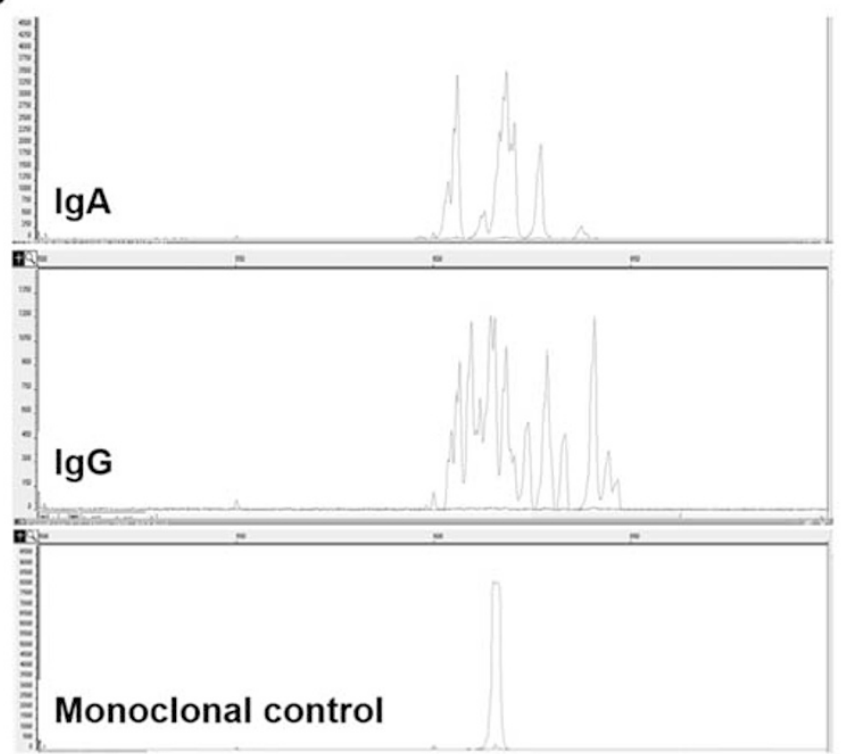

C

\begin{tabular}{|c|c|c|c|c|c|}
\hline \multicolumn{3}{|c|}{ Frozen LN } & \multicolumn{2}{|r|}{ FFPE LN } & \multirow{2}{*}{$\begin{array}{c}\text { Gene Scan Analysis } \\
\text { Conclusion }\end{array}$} \\
\hline status & PC score & IgA-S & PC score & IgA+/CD138+ & \\
\hline $\mathrm{N}+$ & 3 & poly & 3 & - & policlonal \\
\hline $\mathrm{N}+$ & 2 & oligo & 2 & - & IgA restriction \\
\hline $\mathrm{N}+$ & 2 & oligo & 2 & + & IgA restriction \\
\hline $\mathrm{N}+$ & 1 & mono & 2 & + & IgA restriction \\
\hline $\mathrm{N}+$ & 1 & oligo & 3 & + & IgA restriction \\
\hline $\mathrm{N}+$ & 1 & oligo & 1 & + & IgA restriction \\
\hline $\mathrm{N}+$ & 1 & poly & 3 & + & IgM/lgG restriction \\
\hline $\mathrm{N}+$ & 1 & oligo & 2 & + & IgA restriction \\
\hline N- & 2 & oligo & 2 & + & IgA restriction \\
\hline
\end{tabular}

d
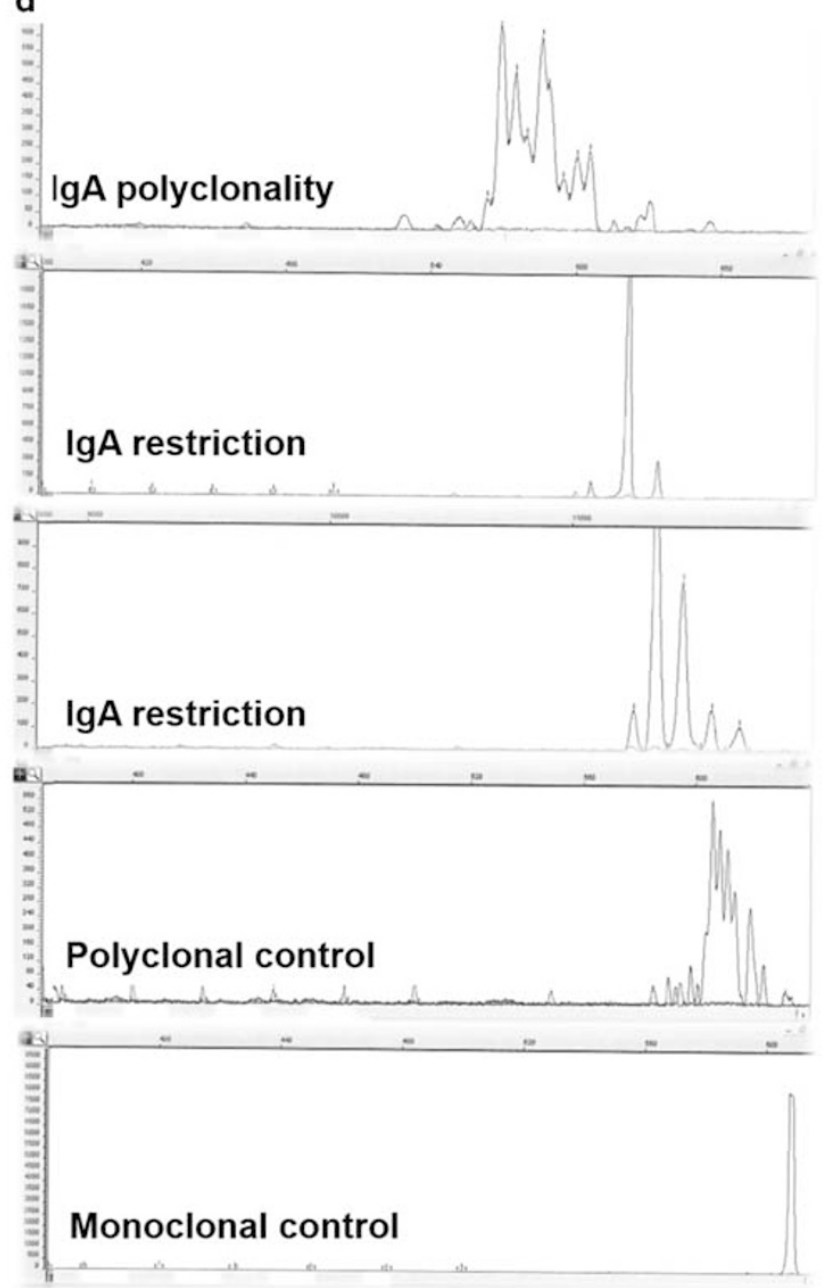

Figure 3 Molecular analysis of Ig genes expressed in PC-rich melanomas. (a) Identification by RT-PCR of the membrane-bound (m) and secreted (s) isoforms of the five Ig isotype families expressed in two primary melanomas with PC infiltration. (b) Immunoscope analysis of IgA-s and IgG-s in one of the two primary melanomas. (c and d) Immunoscope analysis of IgA-s in nine LN draining PC-rich melanomas. FFPE, formalin fixed paraffin embedded; LN, lymph node; $\mathrm{N}-$, uninvolved lymph nodes; $\mathrm{N}+$, lymph nodes involved by the tumor.

IgA PCs was also found in cases with scattered PCs (PC1 score).

Gene Scan analysis, focused on the secretory part of IgA, was performed on frozen material from nine lymph nodes previously studied by immunohistochemistry. Seven of these cases had $10-50 \%$ IgA+ PCs in the lymph node, whereas the other two had no relevant IgA component in the lymph node. In seven out of the nine cases, Gene Scan Analysis showed oligoclonality of the secretory IgA component, suggesting an antigen-driven IgA-immune response (Figures 3c and d). In two out of the nine cases, a lymph node with sheets of PCs (PC3) showed predominance of $\mathrm{IgG}$ PCs and a score 1 case with prominent IgA+ PCs had a polyclonal IgA secretory component. These two cases proved that, even in the presence of a minority of IgA-positive PCs in the lymph node, polyclonality could be detected. Moreover, in the latter case (score 1 with prominent IgA+ PC), a restriction of $\operatorname{IgM}$ and $\operatorname{IgG}$ was 


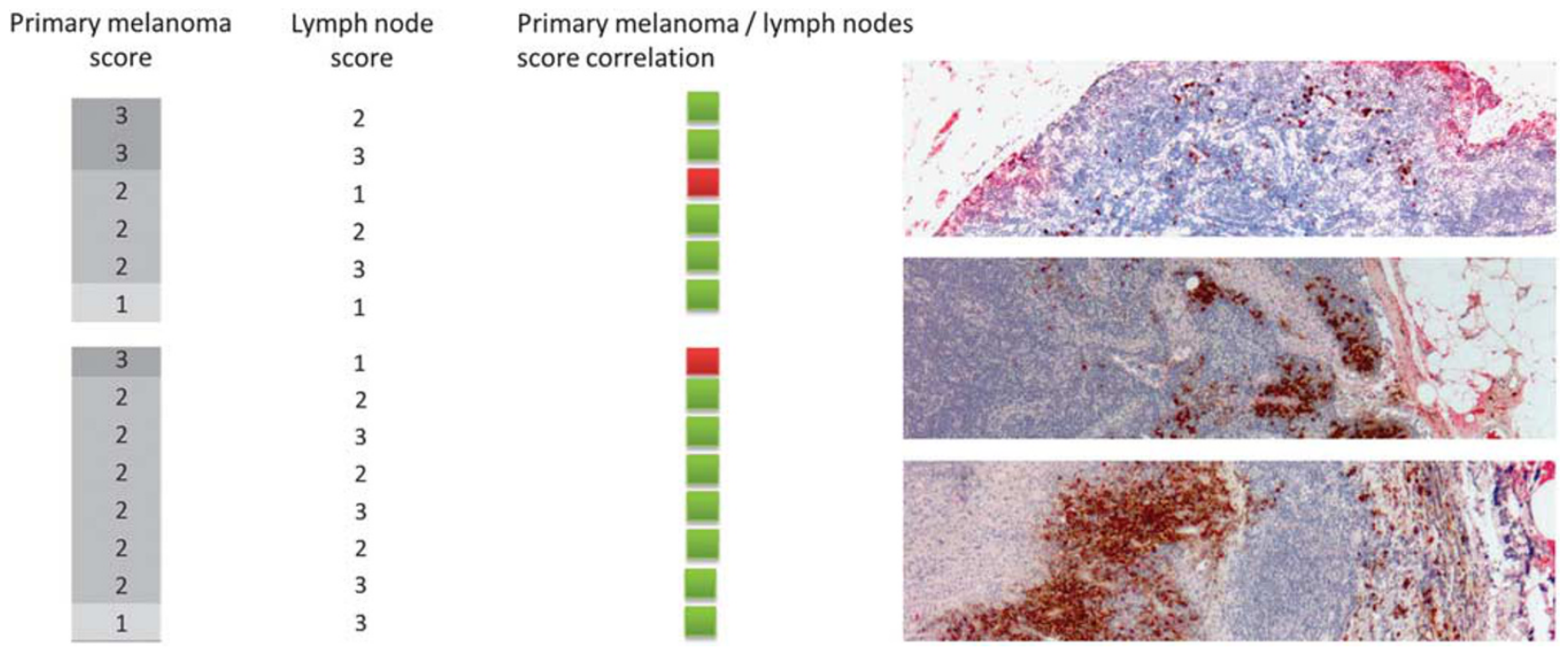

Figure 4 Correlation between the plasma cells' score in primary melanomas and in lymph nodes. After application of the same scoring system used in primary melanomas, a good correlation was found between the amount of plasma cells in the tumoral site and in the draining lymph nodes (pictures on the right, from top to bottom, sparse plasma cells, clusters and sheets in the lymph nodes).

detected, suggesting an antigen-driven immune response involving IgG and IgM, but not IgA.

\section{Outcome of Patients with PC+ Melanomas}

As our data showed that PCs are found in a low percentage of primary melanomas and that they are associated with unfavorable prognostic factors, we analyzed their outcome in a series of 85 thick melanomas from Australia. In this series, PCs were found in 33 cases (38\%), an important increase in comparison to the incidence of $19.5 \%$ of $\mathrm{PC}+$ melanomas that were found among the thick melanomas subgroup from the KUL data set. This could be due to differences between melanomas arising in the Belgian population compared with the Australian one. PC1 melanomas were 12 (36\%), PC2 $11(33 \%)$ and PC3 10 (31\%). In this series of thick melanomas, the presence of PCs correlated again with Breslow thickness $(P=0.0002)$, subtype of melanoma $(P=0.0037)$ and ulceration $(P=0.0002)$. On the other hand, mitotic count and lymphatic/ vascular invasion, associated with the presence of PCs in the original Leuven data set, lost significance in the series from Australia.

Immunohistochemical staining revealed that 21 out of the 33 cases $(70 \%)$ presented a relevant IgA+ PC component. The distribution of the IgA score was homogeneous among the PC scores: also cases with sparse PCs showed predominant expression of IgA. In particular, 9 out of the 12 PC1 melanomas presented between 10 and $50 \%$ of IgA+ PCs in the infiltrate; 6 out of the 11 PC2 melanomas had IgA+ PCs (which in 2 cases comprised $>50 \%$ of the PCs) and 9 out of the 10 PC3 melanomas had in almost all cases $>50 \%$ of IgA+ PCs.
Of the 52 cases without PCs in the inflammatory infiltrate, 26 patients were alive without disease, 3 alive with melanomas, 11 dead owing to melanoma and 12 dead owing to a cause unrelated to melanoma or by unknown cause. Of the 21 PC-rich melanomas (PC2 and PC3), 10 patients were alive without disease, 9 dead owing to melanoma, 1 dead owing to other causes and none alive with melanoma. Of the 12 PC1 melanoma patients, 8 were alive without disease, 2 were alive with melanomas, 1 dead owing to melanoma and 1 dead owing to unknown cause. The Kaplan-Meier curves of survival were not significant for the absolute presence or absence of PCs in the inflammatory infiltrate but were significant for the difference in survival of patients with PC-rich melanomas vs those with melanomas lacking PCs and PC1 melanomas (Figure 5). Hence, patients with PC-rich melanomas showed a worse survival compared with those with $\mathrm{PC}^{\text {neg }}$ melanomas $(P=0.002)$. The survival of patients with PC1 melanomas appeared to be better than that of patients with $\mathrm{PC}^{\text {neg }}$ melanomas, but this finding did not reach statistical significance. The same difference in survival was found when comparing the survival patients with ulcerated melanomas at all sites with that of ulcerated melanomas on the limbs, thereby eliminating the bias of ulceration and site of occurrence (Supplementary Figure S1).

\section{Discussion}

Our work investigated the significance of PCs in melanoma in a wide data set, because in the literature, the role of this B-cell subtype in melanoma is almost unexplored. In fact, only two studies ${ }^{29,30}$ have been performed on this topic $>30$ years ago; one study correlates their presence with an increased 

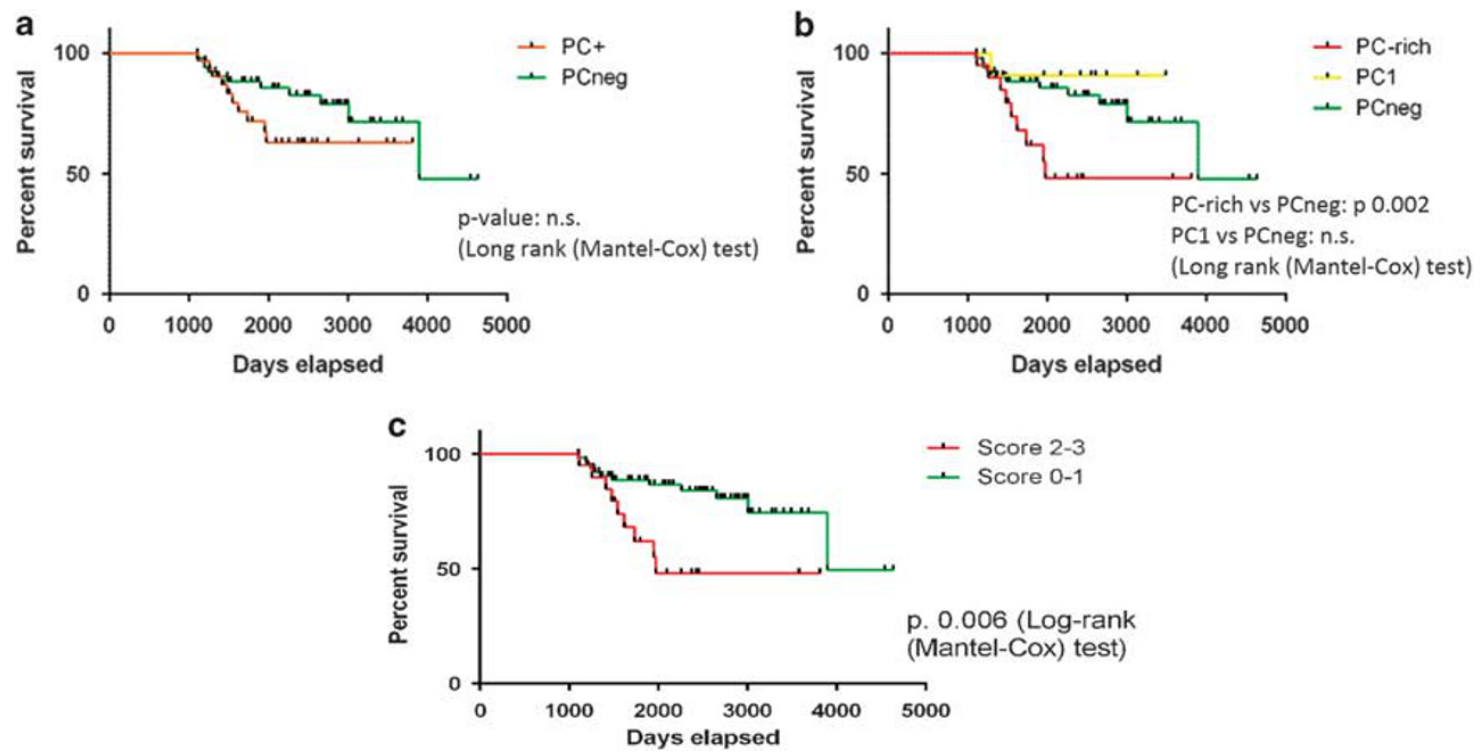

Figure 5 Survival analysis of melanomas with plasma cells. The overall survival does not significantly differ between PC+ and PCmelanomas (a), but among the different PC scores, the score 3 and score 2 melanomas ('PC-rich') show a worse prognosis than score 0 ('PCneg') and score 1 melanomas $(P=0.002)(b)$, therefore these two last groups could be prognostically grouped toghether under the definition of 'PClow' melanomas (c).

risk of lymph node metastasis, and the other study shows an association with poor survival. More recently, a third study has quantified PC at around $8 \%$ of the total cellularity of the inflammatory infiltrate in melanoma metastases. ${ }^{32}$

From our study, it appears that PC+ melanomas are rare and occur in $>4 \%$ of invasive melanomas. From a clinical point of view, PC+ melanomas occur at an older age, ie, some 10 years later than the median age of melanomas lacking PCs. From a histopathological point of view, we found PCs to be associated with features of worse prognosis: high Breslow thickness, high numbers of mitoses, presence of ulceration, and of lymphatic/vascular invasion. Moreover, in the PC-rich group, there was a prevalence of NM, the only melanoma subtype known in the literature to have a worse prognosis compared with the other subtypes. ${ }^{33}$ Ulceration may result in inflammatory infiltrates that contain PCs. To verify that the class switching was related to the tumor and not to the ulceration, we examined also a series of non-neoplastic ulcers and found a low percentage of IgA+ PCs on the total number of PCs in ulcers at non-mucosal sites.

To confirm the poor prognostic significance of PCs among the stromal cells in melanomas, we performed a survival analysis in a selected group of thick melanomas ( $>2 \mathrm{~mm}$ ) to minimize the impact of the thickness on the statistical analysis. In this data set, $38 \%$ of the melanomas carried PCs in the infiltrate. In this 'thick melanomas' subgroup, as expected, the most frequent subtype associated with the presence of PCs was NM. Moreover, PC+ melanomas occurred more frequently in intermittently sun-exposed areas, such as trunk and acral sites, while melanomas lacking PCs involved head and neck and limbs, which are sites known to be chronically sun-damaged. The mere presence of PC in the infiltrate, irrespective of their number, did not, however, predict a poor outcome. This is in contrast with the previous two papers ${ }^{29,30}$ in which PCs were found to correlate with survival, although the relative number of PCs in the infiltrate was not specified. In our survival analysis, only PC-rich (PC2-PC3) melanomas, ie, those showing clusters and confluent sheets of PCs, showed a worse survival compared with $\mathrm{PC}^{\text {neg }}$ melanomas, while patients with PC1 melanomas (showing scattered PCs in the stromal infiltrate) appeared to have a better survival than those lacking PCs, although no statistical significance was reached. Therefore, we can divide cases into two groups according to survival, the PC-rich melanomas (PC2-PC3), and the PC-low melanomas (PC1-PC0) (Figure 5c). The influence of the quantity of plasma cells on survival could be due to the fact that, at the beginning of the plasma cell response, PCs may either have a beneficial effect or their effect is not yet detectable, because it is counterbalanced by other immune mechanisms.

When the isotype of the PCs was explored, we found that the two most predominant immunoglobulins produced are IgG and IgA. In general, the presence of tumor-specific antibodies has always been regarded as a good prognostic factor. ${ }^{34}$ The presence of tumor-infiltrating B cells and Ig $\kappa$ chains genes' overexpression within the tumor bed have been positively correlated with metastasis-free survival and response to chemotherapy in breast cancer. ${ }^{31}$ On the other hand, 
different immunoglobulin isotypes have been shown to have different roles in human cancers. IgM and IgD identify the naive, preswitched $\mathrm{B}$ cell. The lifespan of this cell depends on exposure to antigen: according to Burnet's 'clonal selection' theory, $B$ cells are rescued from apoptosis by their response to antigens. ${ }^{35} \mathrm{~B}$ cells can respond directly to an antigen, independently from $\mathrm{T}$ cell help, or can participate in cognate recognition of the antigen with $\mathrm{T}$ cells, through the costimulatory molecules CD80 and CD86. The latter takes place during a germinal center reaction and results in class switching and somatic hypermutations of the immunoglobulin variable domains, thus giving birth to Ig variants with various affinities for the antigen, thereby improving its recognition. ${ }^{35}$ In this manner, $B$ cells differentiate into PCs and switch from expression of the constant region of the $\operatorname{IgM}(\mathrm{C} \mu)$ to expression of the same VHDJH-exon with any of the downstream $\mathrm{CH}$ genes $(\mathrm{C} \alpha 1-2, \mathrm{C} \gamma 1-4$ or $\mathrm{C} \varepsilon$ ), thereby becoming an IgA, IgG or IgE molecule. Class switching appears to be dependent on external cytokine signals: IFN- $\gamma$ favors IgG2 switching, IL-4 favors IgG4 and IgE switching, and transforming growth factor- $\beta$ (TGF- $\beta$ ) favors IgA switching. ${ }^{35}$ In our series of $\mathrm{PC}+$ melanomas, we found predominantly IgG and IgA expression in the PCs. With regard to IgG, the IgG4 subclass has been linked to a worse outcome and has turned out to have blocking effects on the binding of tumor-specific IgG1. ${ }^{36}$ On the other hand, the ectopic expression of Fc $\gamma$ RIIB, an inhibitory receptor for IgG, on melanoma cells was found to promote liver metastasis allowing the tumor to escape humoral immunity. ${ }^{15}$

Unexpectedly, a high proportion of PCs expressed IgA. IgA has been found to have blocking properties on cytotoxic $\mathrm{T}$-cell reactions against melanoma ${ }^{37}$ and has been shown to have a tolerogenic role in the gut $^{38}$ and an immunosuppressive role counteracting IgG activities in intestine transplantation. ${ }^{39}$ More recently, Shalapour et $a l^{40}$ correlated IgA-producing plasmablasts with the failure of immunogenic chemotherapy in mice with prostatic cancer, confirming the role of $\operatorname{IgA}$ in counteracting the immune response.

In our data set, the relative number of IgA + PCs tended to increase with the total number of PCs (expressed as PC score) as well as with melanoma thickness. Indeed, in the 'thick melanomas' data set, IgA+ PCs were more frequently found than in the original data set. Moreover, at the Gene Scan Analysis, transcripts of few different lengths were found for IgA indicating oligoclonality, whereas transcripts with high variability in length were found for IgG, indicating polyclonality. This strongly suggests an antigen-driven restriction of the tumor-associated IgA+ PCs. The nature of this antigen is as yet unknown but currently under study.

Finally, we sought to elucidate the origin of the melanoma-associated IgA+ PCs. Under normal circumstances, B lymphocytes are not detectable in the skin owing to their very low number. ${ }^{41}$ The adaptive humoral immune response classically originates in secondary lymphoid organs (SLO) but may be generated in situ within TLS. TLS are inducible and transient structures that occur in non-lymphoid organs, and that share with SLO the same cytokines involved in their genesis, and the same B and T topography. ${ }^{22}$ TLS are composed of T-areas containing mature dendritic cells and $\mathrm{B}$-areas containing germinal centers with evidence of isotype switching in B cells; in addition, TLS possess PNAd+HEV and express a range of cytokines important for the organization of SLO. ${ }^{42}$ Not all these features are present at the same time, suggesting evolution over time of TLS. ${ }^{31}$ A recent study in lung carcinomas demonstrated that TLS were able to produce polyclonal IgG and IgA directed against several tumor antigens, ${ }^{31}$ and in melanoma metastases, B cells in the follicles of TLS were shown to be involved in IgA responses. ${ }^{43}$ However, analysis of our series showed TLS in only 2 of the 15 primary melanomas; hence, it is unlikely that the PC were produced 'in situ' in the skin.

After contact with the antigen and their phenotype switching in Peyer's plaques, intestinal plasmablasts migrate to the lymph nodes in order to proliferate and hypermutate; thereafter, they finally reach the lamina propria to mature into IgA-secreting PCs. ${ }^{44}$ Similarly, B cells continuously recirculate between skin and lymph nodes. ${ }^{45}$ Therefore, we investigated the loco-regional lymph nodes draining the $\mathrm{PC}+$ melanoma site. In these lymph nodes, we generally observed a considerable number of IgA+ PCs, the number of which correlated with the number in the primary melanoma. This suggests that the IgA response involves a recirculation of $\mathrm{IgA}+\mathrm{PCs}$ (or plasmablasts) between the skin and lymph nodes. Finally, the Gene Scan Analysis performed on the DNA extracted from the loco-regional lymph nodes showed oligoclonality of the secretory IgA component both in the involved and uninvolved tumor-draining lymph nodes, thereby indicating that the IgA + PCs in the lymph node also showed antigen restriction. This result is in agreement with the finding of oligoclonal patterns in T-cell receptor rearrangements in both primary melanomas and draining lymph nodes. ${ }^{45}$

In conclusion, primary melanomas with clusters and sheets of PCs in the inflammatory micro-environment are rare $(3.7 \%)$, occur in older patients and are associated with histological markers of poor prognosis and with a poor survival. Melanoma-infiltrating PCs are predominantly of the IgG and IgA isotypes. The low incicence of PC-positive cases and their already high-risk nature limit the utility of these findings in prognostication, and anyway, additional validation would be required before using the findings for clinical decision-making purposes. Nevertheless, we suggest that these IgAs, which are likely antigen-induced, exert a tumor-promoting role, either by interaction 
with other cells in the micro-environment or by blocking soluble, growth-inhibiting molecules. Characterization of these IgA and determination of their target antigens may help to understand the role of humoral immunity in melanoma.

\section{Acknowledgments}

FMB is funded by the MEL-PLEX research training programme ('Exploiting MELanoma disease comPLEXity to address European research training needs in translational cancer systems biology and cancer systems medicine', Grant agreement no: 642295, MSCA-ITN-2014-ETN, Project Horizon 2020, in the framework of the MARIE SKŁODOWSKA-CURIE ACTIONS). JW received funding as an Experienced Researcher within the Marie Curie Industry-Academia Partnership and Pathways programme, SYS-MEL, which is funded under the FP7-PEOPLE-2013-IAPP scheme (Grant agreement number 611107) and is currently funded from the Melanoma Research Alliance (Team Science Research Award; USA).

\section{Disclosure/conflict of interest}

The authors declare no conflict of interest.

\section{References}

1 Bastian BC. The molecular pathology of melanoma: an integrated taxonomy of melanocytic neoplasia. Annu Rev Pathol 2014;9:239-271.

2 Bae JM, Choi YY, Kim DS et al. Metastatic melanomas of unknown primary show better prognosis than those of known primary: a systematic review and metaanalysis of observational studies. J Am Acad Dermatol 2015;72:59-70.

3 Lo JA, Fisher DE. The melanoma revolution: from UV carcinogenesis to a new era in therapeutics. Science 2014;346:945-949.

4 Powles T, Eder JP, Fine GD et al. MPDL3280A (anti-PDL1) treatment leads to clinical activity in metastatic bladder cancer. Nature 2014;515:558-562.

5 Herbst RS, Soria JC, Kowanetz M et al. Predictive correlates of response to the anti-PD-L1 antibody MPDL3280A in cancer patients. Nature 2014;515: 563-567.

6 Tumeh PC, Harview CL, Yearley JH et al. PD-1 blockade induces responses by inhibiting adaptive immune resistance. Nature 2014;515:568-571.

7 Sharma P, Allison JP. Immune checkpoint targeting in cancer therapy: toward combination strategies with curative potential. Cell 2015;161:205-214.

8 Zhou D, Huang C, Lin Z et al. Macrophage polarization and function with emphasis on the evolving roles of coordinated regulation of cellular signaling pathways. Cell Signal 2014;26:192-197.

9 Martinez FO, Gordon S, Locati M et al. Transcriptional profiling of the human monocyte-to-macrophage differentiation and polarization: new molecules and patterns of gene expression. J Immunol 2006;177: 7303-7311.

10 Biswas SK, Mantovani A. Macrophage plasticity and interaction with lymphocyte subsets: cancer as a paradigm. Nat Immunol 2010;11:889-896.

11 Zitvogel L, Kroemer G. CD103+ dendritic cells producing interleukin-12 in anticancer immunosurveillance. Cancer Cell 2014;26:591-593.

12 Balkwill FR, Capasso M, Hagemann T. The tumor microenvironment at a glance. J Cell Sci 2012;125: 5591-5596.

13 Ladányi A. Prognostic and predictive significance of immune cells infiltrating cutaneous melanoma. Pigment Cell Melanoma Res 2015;28:490-500.

14 Kitamura T, Qian BZ, Pollard JW. Immune cell promotion of metastasis. Nat Rev Immunol 2015;15: 73-86.

15 Smith HA, Kang Y. The metastasis-promoting roles of tumor-associated immune cells. J Mol Med (Berl) 2013;91:411-429.

16 Bulman A, Neagu M, Constantin C. Immunomics in skin cancer - improvement in diagnosis, prognosis and therapy monitoring. Curr Proteomics 2013;10:202-217.

17 Clemente CG, Mihm MC Jr, Bufalino R et al. Prognostic value of tumor infiltrating lymphocytes in the vertical growth phase of primary cutaneous melanoma. Cancer 1996;77:1303-1310.

18 Clark WH Jr, Elder DE, Guerry DT et al. Model predicting survival in stage I melanoma based on tumor progression. J Natl Cancer Inst 1989;81: 1893-1904.

19 Cipponi A, Wieers G, van Baren $\mathrm{N}$ et al. Tumor-infiltrating lymphocytes: apparently good for melanoma patients. But why? Cancer Immunol Immunother 2011;60:1153-1160.

20 Hillen F, Baeten CIM, van de Winkel A et al. Leukocyte infiltration and tumor cell plasticity are parameters of aggressiveness in primary cutaneous melanoma. Cancer Immunol. Immunother 2008;57:97-106.

21 Hussein MR, Elsers DAH, Fadel SA et al. Immunohistological characterization of tumour infiltrating lymphocytes in melanocytic skin lesions. J Clin Pathol 2006;59:316-324.

22 Fridman WH, Remark R, Goc J et al. The immune microenvironment: a major player in human cancers. Int Arch Allergy Immunol 2014;164:13-26.

23 Linnebacher M, Maletzki C. Tumor-infiltrating B cells: The ignored players in tumor immunology. Oncoimmunology 2012;1:1186-1188.

24 Linnebacher M. Tumor-infiltrating B cells come into vogue. World J Gastroenterol 2013;19:8-11.

25 Martinez-Rodriguez M, Thompson AK, Monteagudo C. A significant percentage of CD20-positive TILs correlates with poor prognosis in patients with primary cutaneous malignant melanoma. Histopathology 2014;65:726-728.

26 Fremd C, Schuetz F, Sohn C et al. B cell-regulated immune responses in tumor models and cancer patients. Oncoimmunology 2013;2:e25443.

27 Ladányi A, Kiss J, Mohos A et al. Prognostic impact of B-cell density in cutaneous melanoma. Cancer Immunol Immunother 2011;60:1729-1738.

28 Bruno TC, French JD, Jordan KR et al. Influence of human immune cells on cancer: studies at the University of Colorado. Immunol Res 2013;55:22-33.

29 Weissmann A, Roses DF, Harris MN et al. Prediction of lymph node metastases from the histologic features of 
primary cutaneous malignant melanomas. Am J Dermatopathol 1984;6:35-41.

30 Mascaro JM, Molgo M, Castel T et al. Plasma cells within the infiltrate of primary cutaneous malignant melanoma of the skin. A confirmation of its histoprognostic value. Am J Dermatopathol 1987;9:497-499.

31 Germain C, Gnjatic S, Dieu-Nosjean MC. Tertiary lymphoid structure-associated B cells are key players in anti-tumor immunity. Front Immunol 2015;23:6-67.

32 Erdag G, Schaefer JT, Smolkin ME et al. Immunotype and immunohistologic characteristics of tumorinfiltrating immune cells are associated with clinical outcome in metastatic melanoma. Cancer Res 2012;72: 1070-1080.

33 Bolognia JL, Jorizzo JL, Schaffer JV. Melanoma. In: Jean LB, Joseph LJ, Julie VS (eds). Dermatology, 3rd (edn). Elsevier: St Louis, USA, 2012, pp 922.

34 Morton D, Eilber FR, Malmgren RA et al. Immunological factors which influence response to immunotherapy in malignant melanoma. Surgery 1970;68:158-163.

35 Vale AM, Schroeder HW Jr. Clinical consequences of defects in B-cell development. J Allergy Clin Immunol 2010;125:778-787.

36 Karagiannis $\mathrm{P}$, Gilbert AE, Josephs $\mathrm{DH}$ et al. IgG4 subclass antibodies impair antitumor immunity in melanoma. J Clin Invest 2013;123:1457-1474.

37 O'Neill PA, Romsdahl MM. IgA as a blocking factor in human malignant melanoma. Immunol Commun 1974;3:427-438.
38 Doi T, Kanai T, Mikami Y et al. IgA plasma cells express the negative regulatory co-stimulatory molecule programmed cell death 1 ligand and have a potential tolerogenic role in the intestine. Biochem Biophys Res Commun 2012;425:918-923.

39 Ningappa M, Ashokkumar C, Ranganathan S et al. Mucosal plasma cell barrier disruption during intestine transplant rejection. Transplantation 2012;94: 1236-1242.

40 Shalapour S, Font-Burgada J, Di Caro G et al. Immunosuppressive plasma cells impede T-cell-dependent immunogenic chemotherapy. Nature 2015;521:94-98.

41 Egbuniwe IU, Karagiannis SN, Nestle FO et al. Revisiting the role of $\mathrm{B}$ cells in skin immune surveillance. Trends Immunol 2015;36:102-111.

42 Dieu-Nosjean MC, Goc J, Giraldo NA et al. Tertiary lymphoid structures in cancer and beyond. Trends Immunol 2014;35:571-580.

43 Cipponi A, Mercier M, Seremet T et al. Neogenesis of lymphoid structures and antibody responses occur in human melanoma metastases. Cancer Res 2012;72: 3997-4007.

44 Hahn A, Thiessen N, Pabst R et al. Mesenteric lymph nodes are not required for an intestinal immunoglobulin A response to oral cholera toxin. Immunology 2010;129:427-436.

45 Thurin M, Marincola FM. Molecular diagnostics for melanoma: methods and protocols. In: Magdalena T, Francesco MM (eds). Methods in Molecular Biology, Springer: New York, USA, 2014, pp 287-324.

Supplementary Information accompanies the paper on Modern Pathology website (http://www.nature.com/ modpathol) 